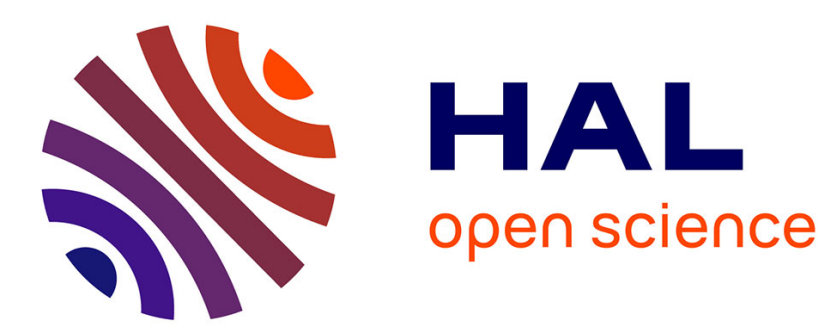

\title{
Différences, talents et utopies. Apologie de John Rawls, derechef
}

Alain Boyer

\section{To cite this version:}

Alain Boyer. Différences, talents et utopies. Apologie de John Rawls, derechef. Les études philosophiques, 2020, N²04 (4), pp.5-27. 10.3917/leph.204.0005 . hal-03180893

\section{HAL Id: hal-03180893 \\ https://hal.sorbonne-universite.fr/hal-03180893}

Submitted on 25 Mar 2021

HAL is a multi-disciplinary open access archive for the deposit and dissemination of scientific research documents, whether they are published or not. The documents may come from teaching and research institutions in France or abroad, or from public or private research centers.
L'archive ouverte pluridisciplinaire HAL, est destinée au dépôt et à la diffusion de documents scientifiques de niveau recherche, publiés ou non, émanant des établissements d'enseignement et de recherche français ou étrangers, des laboratoires publics ou privés. 


\section{Différences, talents et utopies. Apologie de John Rawls,} derechef.

L'œuvre de Rawls est constituée de trois piliers, dont on n'étudiera (partiellement) ici que le premier $^{1}$, celui constitué de l'opus maximum, la Théorie de la Justice $(T J, 1971)^{2}$ et de sa reformulation, rédigée près de vingt ans plus tard (Justice as Fairness) $)^{3}$. La TJ, dès sa parution, provoqua un raz de marée inattendu de publications, élogieuses et critiques, lesquelles ont fini par donner lieu à un total renouvellement et une résurrection de la philosophie politique de langue anglaise. II y aura pour toujours un avant et un après la TJ. Ayant publié plusieurs articles sur elle depuis 1988, j'ai entrepris, au début de l'année 2016, de consacrer un ouvrage à la critique de quelques-unes des plus célèbres objections qui lui ont été adressées. II eût été impossible de tenter de répondre à toutes les objections, étant donné leur nombre astronomique. II

\footnotetext{
${ }^{1}$ Le deuxième moment de l'œuvre commence en 1985 et aboutit au second ouvrage du maître : Libéralisme politique (1993), trad. Puf (LP); le troisième, plus modeste, et moins applaudi, est constitué par The Law of Peoples, 1999, trad. Paix et démocratie, La Découverte, 2006. On doit consulter également les Collected Papers, Harvard, 1999 ; les précieux cours posthumes d'histoire de la philosophie morale et ceux d'histoire de la philosophie politique (Harvard), ainsi que la thèse de théologie du tout jeune Rawls, découverte à Princeton après sa mort : Le Péché et la foi, Hermann, 2010. Je remercie très vivement Jean-Cassien Billier d'avoir organisé une journée d'études autour de mon livre Apologie de John Rawls, Puf, 2018, en Sorbonne, le 08 février 2020, et tous les participants. 2 Seconde édition révisée, Oxford, 1999 ; c'est cette version, rédigée en 1973, qui a servi à toutes les traductions, telle la française (Seuil, 1987).

${ }^{3}$ JF ; trad. La Justice comme équité, La Découverte, 2003.
} 
$s^{\prime}$ agit uniquement de certaines objections du libertarien minarchiste ${ }^{4}$ Robert Nozick, dans son ouvrage très original Anarchy, State and Utopia $^{5}$, du " communautarien » Michael Sandel, dans son pamphlet contre Rawls, Le Libéralisme et les limites de la Justice ${ }^{6}$, mais aussi, plus indirectement, de celles de l'adversaire utilitariste, le Prix Nobel d'économie John Harsanyi, ou encore celles du marxiste G. A. Cohen $^{7}$. Je vais ici reformuler quelques-unes des idées soutenues dans le livre, en y ajoutant de nouvelles pistes et apportant des corrections. Je n'aborderai essentiellement que les objections de Nozick ${ }^{8}$, et ajouterai de nouveaux éléments concernant l'univocité du principe de différence (PD) et les modes de production compatibles avec sa réalisation selon Rawls.

Se défendre contre des objections, comme le fit Descartes à l'initiative du Père Mersenne, se dit en grec "apologeïn »: parler pour écarter, "lever" (apo) une accusation, en montrant son absence de fondement dans la vie (Socrate) ou dans les écrits de celui qui se défend. En Grèce, l'accusé y procède lui-même, et Apulée

\footnotetext{
${ }^{4}$ Anti-anarchiste, et partisan du laisser-faire accompagné d'un État minimal, absolument pas redistributeur. II serait intéressant de comparer le minarchisme, l'anarcho-capitalisme et ce que Nietzsche appelait le misarchisme (Généalogie de la Morale, III).

${ }^{5}$ Cambridge, 1982 ; trad. Seuil, 1999.

${ }^{6}$ Dont je m'étonne que JP Dupuy puisse dire qu'il reste " l'une des meilleures introductions à l'œuvre du maître de Harvard ", in Préface à Sandel M., Ce que l'argent ne saurait acheter, Seuil, 2014.

${ }^{7}$ Rescuing Justice and Equality, Harvard, 2008.

${ }^{8}$ Entièrement reprises en 1982 par Sandel, qui y ajoute des arguments contre le "Self " rawlsien, "premier par rapport à ses fins ", puis, après $L P$, une accusation infondée, selon laquelle, la théorie de la justice étant "politique et non métaphysique ", serait de ce fait " non morale ». Voir les ch. VI et VII de I'Apologie.
} 
le fera encore par écrit. Je me suis permis de défendre Rawls contre ses accusateurs, car il ne l'a guère fait lui-même, sauf discrètement, en note. Et c'est donc une Apologie de John Rawls qui a paru aux Puf en 2018. L' objectif principal était le suivant: à supposer que la critique rawlsienne de la notion (ou de l'absence de la notion) de la personne dans la pensée utilitariste soit fondée, est-il légitime, étant donné la caractérisation par Rawls de son "principe de différence ", de retourner sa propre critique anti-utilitariste contre lui, en arguant que lui non plus ne permet pas de fonder une notion concrète et complexe de personne, ne laissant au lecteur qu'un pseudo-soi, purifié, hyper-abstrait, nouménal, détaché et désencastré de tout, une personne sans qualités, sans talents, aussi peu épaisse que la pseudo-personne utilitariste justement dénoncée par l'auteur de la TJ lui-même ? II s'agit donc de prendre au sérieux et de réfuter un argument de type Tu Quoque!, lequel retourne contre un argumentateur l'un de ses arguments critiques, en montrant que, malgré qu'il en eût, il tombe lui-même sous la critique qu'il a émise contre une tierce position. Cette stratégie argumentative, légitime, a été utilisée contre la $T J$ d'abord par Nozick, puis reprise et approfondie par le "communautarien" républicain anti-libéral Michael Sandel, et considérée comme valide par de nombreux auteurs. L'Apologie tente donc de répondre à cette accusation, et l'acquittement de John Rawls est proposé au lecteur. 
La pensée du maître de Harvard nous fournit l'un des seuls systèmes pratiques, éthico-politiques, du siècle dernier. Ce système (partiel) est lui-même le corrélat ou le produit d'une méthode de choix « contractualiste ", la " position originelle » (PO), où les agents rationnels qui ont à préférer une conception de la justice sociale sont soumis à un "voile d'ignorance " (VI) quant à ce qui les singularise, et à quelques autres contraintes informationnelles et formelles. Le centre du système est, quant à lui, composé d'une certaine conception particulière de la justice sociale, dont Rawls tente de montrer qu'elle sera préférée dans la PO à toute une liste d'autres théories possibles, dont l'utilitarisme. Tout devrait se passer comme si cette conception était « la meilleure réponse possible de chacun aux exigences des autres ${ }^{9}$. Elle est organisée hiérarchiquement, lexicalement, en deux (de fait trois) principes de justice. Ces principes

\footnotetext{
9 TJ, § 20 ; cette section peut évoquer, me semble-t-il, aux yeux des économistes, le théorème d'Arrow-Debreu sur l'équilibre walrasien comme optimum de Pareto (par. 3), et même, je crois, dans la phrase citée (par. 2), le théorème de Nash (tout jeu fini a au moins une solution, en utilisant au besoin des stratégies mixtes). Si je "joue " les principes de Rawls, et que je m'aperçois après coup que les autres ont fait de même, je n'aurai pas à regretter mon choix (équilibre de Nash, curieusement supposé unique). Il ne s'agit bien sûr que d'une analogie, car même si nous devons "aspirer à une géométrie morale ", les principes de justice étant alors strictement déduits de la description idoine de la PO, Rawls admet que cet idéal, qu'il faut cependant avoir à l'esprit, ne sera pas accompli, bien des éléments de la procédure dépendant de nos intuitions. L'allusion aux jeux (non coopératifs et à somme variable: la coopération peut rapporter plus que la non-coopération égoïstement rationnelle) indique que la PO n'est pas une situation de délibération commune. Chacun choisit seul : le pari théorique est que tous choisiront les deux principes de justice proposés par Rawls. Rien ne leur permet de raisonner de manière différente, ni de profiter d'une information pour tenter d'obtenir un avantage par la menace ou la négociation. La PO n'est pas non plus de fait un " jeu ", et il ne s'agit pas de "stratégies", d'où le caractère très allusif de ce passage. Mais il est clair que Rawls aurait aimé pouvoir produire un « théorème ".
} 
publics régulateurs de la coopération sociale sont ceux d'une "société bien ordonnée ", considérée comme un système de coopération équitable entre citoyens libres et égaux d'une démocratie constitutionnelle moderne.

Plusieurs conceptions monistes, constituées d'un seul principe, se présentent immédiatement comme de bonnes candidates à ce

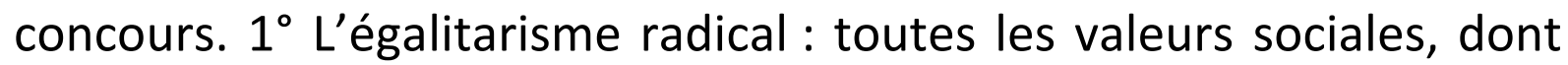
la richesse, doivent être réparties ou distribuées de manière strictement égale, point. $2^{\circ}$ l'utilitarisme : il faut préférer un état social faisable, réalisable, qui maximise la quantité de bonheur (ou de satisfaction des désirs), éventuellement divisée par le nombre d'individus concernés si la population augmente (utilitarisme de la moyenne $)^{10} \cdot 3^{\circ}$ le perfectionnisme : il faut maximiser la probabilité que puissent s'épanouir des personnalités à la vertu ou à l'excellence la meilleure. $4^{\circ}$ Le libéralisme pur ou "libertarisme ${ }^{11}$ ne propose pas de description de l'état final auquel son principe unique de liberté ou propriété de soi peut conduire : il lui suffit de dire qu'il est

\footnotetext{
${ }^{10}$ Par ailleurs, si tout le monde a la même fonction d'utilité, la baisse de l'utilité marginale de l'argent favorisera l'égalité de richesse, laquelle maximise l'utilité (un euro de moins pour un millionnaire affecte négativement moins ce dernier qu'il n'affecte positivement le SDF à qui il est donné, etc, jusqu'à la répartition 50/50). Beaucoup d'utilitaristes, en particulier les économistes tel Harsanyi, sont réticents quant à l'hypothèse d'identité des fonctions d'utilité et ne présentent donc pas cet argument qui rend l'utilitarisme l'égalitariste, aspect qui ne serait du reste qu'une conséquence inattendue de la théorie, et non l'un de ses principes.

${ }^{11}$ II y a des différences entre le libéralisme classique et le libertarisme moderne, mais elles ne nous concernent pas ici. Rawls (§ 12), citant Smith, se contente de parler de « système de la liberté naturelle $"$. Le libertarisme est plus radicalement anti-étatiste que le libéralisme classique, en général. Certains libertariens actuels, tels Hans-Hermann Hoppe, accusent fortement Hayek d'étatisme, injure suprême.
} 
Pareto-optimal, en ce sens qu'aucune réallocation des ressources ne permettrait d'obtenir un état où aucune personne ne verrait ses ressources diminuer, alors qu'au moins l'une d'elles verrait sa situation s'améliorer. Le marché libre est capable de produire des états Pareto-optimaux ${ }^{12}$. Mais il existe une infinité d'états Paretooptimaux incomparables, et tous possiblement issus de processus " historiques " d'acquisition et de transferts justes, consentis, des biens et des services. Comme l'utilitarisme, le libertarisme du "laisser-faire", même sous la contrainte morale que tous les échanges soient honnêtes, est compatible avec de très grandes disparités sociales.

II semble clair qu'aux yeux de Rawls, toutes les théories monistes se heurtent à l'un de nos jugements intuitifs réfléchis. Ainsi, l'utilitarisme autorise que l'un des individus voit sa satisfaction baisser, si cette baisse est plus que compensée par la hausse de la somme des satisfactions des autres: il lui est " sacrifié » ". II faut une conception pluraliste. C'est ce que propose le pluralisme "intuitionniste », au sens de Ross: on peut ainsi attacher une certaine valeur à l'égalité, et une autre à la maximisation de la quantité de bien-être, ce qui peut être représenté de manière géométrique par la méthode micro-économique des

\footnotetext{
${ }^{12}$ Si la concurrence est parfaite et qu'il n'y a pas d'externalités, et donc des marchés pour tout.
} 
"courbes d'indifférence ${ }^{13}$. Chacun peut établir sa manière de " pondérer » ces deux principes prima facie ${ }^{14}$ intuitifs, mais tirant dans des directions différentes. Si tout le monde les pondérait de la même manière, il n'y aurait pas de problème: un méta-critère éthique commun à tous permettrait de tracer les mêmes courbes d'indifférence, ne se coupant jamais, chaque point sur une courbe plus au Nord-Est qu'une autre étant préféré aux points de toute courbe "inférieure ". Ceux qui attribuent peu ou prou la même valeur aux deux principes intuitifs se retrouvent avec les courbes bien connues de ceux des consommateurs qui «préfèrent les mélanges ». Mais, et c'est une thèse forte, Rawls nie qu'il existe un tel consensus moral sur le sous-pesage correct des valeurs sociales en question. Certains privilégieront le bien-être total, ne donnant à l'égalité qu'une place plus mesurée : leurs courbes d'indifférence seront plus verticales (si le bien-être est en abscisse) que celles du précédent ${ }^{15}$. Or, tout point qui est au Nord-Est d'un autre est préféré à celui-ci : D sera préféré par le second évaluateur (le plus " welfariste ») à un point $\mathrm{C}$ sur une courbe plus au sud-ouest, alors que le premier décideur sera indifférent entre $C$ et $D$, points où sa courbe d'indifférence (en continu) croise celles du second (en tirets). Si l'on

\footnotetext{
${ }^{13}$ TJ, § 7, fig. 1 et 2 ; Apologie, fig. p. 56.

${ }^{14}$ Ross (traducteur d'Aristote, par ailleurs) aurait peut-être dû employer plutôt l'expression " pro tanto "; il s'agit de principes (ou de devoirs) qui sont de prime abord acceptables de manière absolue, mais qui peuvent en fait être contrecarrés par d'autres principes. Par exemple, tenir ses promesses : cf. mon Chose Promise, Puf, 2014.

${ }^{15} \mathrm{TJ}$, ibid., fig. 2. Il faut avoir ces figures sous les yeux pour comprendre les raisonnements qui les commentent.
} 
veut « agréger » les deux jugements, on obtiendra une « fonction de choix social ", si l'on m'autorise à parler comme Arrow, incohérente : D est à la fois " socialement » (en l'occurrence, à deux) préféré à C (les deux points sont sur des courbes d'indifférence différentes du second évaluateur), lequel point est par ailleurs indifférent avec D (car sur une même courbe d'indifférence du premier). Du point de vue collectif, $D$ est donc à la fois (strictement) préféré à $C$ et indifférent avec lui. Incohérence. La seule solution, si je puis me permettre de poursuivre l'analogie arrovienne, serait un dictateur intuitionniste, les pondérations " sociales » étant toujours identiques aux siennes. Le pluralisme intuitionniste ne saurait fonctionner au niveau social et constitutionnel d'une démocratie pluraliste ${ }^{16}$.

La seule possibilité qui demeure si l'on veut échapper aux monismes contre-intuitifs et aux intuitionnismes pluralistes socialement incohérents, c'est le pluralisme hiérarchisé, ou « lexical ${ }^{17}$. Le premier principe rawlsien est celui, tout kantien, des droits et libertés égales maximales compatibles avec le fait que tous

\footnotetext{
${ }^{16}$ Le point $\mathrm{D}$ comme "principe de différence ", en revanche, pourra être vu comme un "point focal» (au sens de Thomas Schelling) entre l'égalité et l'efficacité comme le suggérera l'économiste $E$. Phelps.

17 Dont je crois pouvoir donner un illustre exemple (ch. III, p. 125, not 1) dans la théorie pascalienne des trois ordres. Le plus grand théorème d'Archimède, prince de l'ordre de l'esprit, ne saurait compenser le moindre manque de charité, dont l'ordre a pour prince JC. Mais le plus petit théorème l'emporte infiniment sur tous les éclats de l'ordre des corps. L'ordre lexical est ... non archimédien (on devrait dire " eudoxien »). De plus, les principes rawlsiens sont ordonnés comme une suite de maximisations contraintes. Ce n'est que si la maximisation d'un principe antérieur est théoriquement atteinte, que l'on peut enclencher le principe suivant. L'ordre lexical est très rigoureux, "dogmatique ", comme le lui ont reproché des économistes importants, tels Harsanyi et Arrow, et ne saurait être appliqué sans réflexion dans tous les cas. Esprit de finesse.
} 
les citoyens aient les mêmes, et dont le droit à l'intégrité de la personne est l'un des plus importants; le second principe est luimême lexical, sa seconde partie ne pouvant s'opposer aux exigences de la première: $1^{\circ} L^{\prime}$ égalité réelle des chances; $2^{\circ}$ le principe de différence (PD) : toute inégalité de pouvoir, de fortune ou de revenu doit être au bénéfice de tous, et fonctionner au plus grand bénéfice possible de ceux et celles qui en ont le moins. L'égalité "réelle » (fair) des chances est cruciale : les libéraux classiques, tel Hayek, applaudissent le principe (formel) des «carrières ouvertes aux talents", cet aspect "méritocratique » étant même fondamental dans le processus d'émancipation libéral des « entraves » de l'Ancien Régime, irrationnel en ceci qu'il se privait sans raison des talents potentiels de ceux et celles qui ne sont pas « nobles ${ }^{18}$. Mais elle est insuffisante, car le lieu social de naissance, et l'éducation familiale ont aussi des effets considérables sur les chances effectives, les opportunités réelles des enfants semblablement talentueux et motivés: ils n'ont pas les mêmes chances de pouvoir développer leurs talents. Les socialistes ont eu raison de critiquer le libéralisme classique sur ce point. II faut donc que l'État, par exemple via des politiques fortes d'éducation publique, et de bourses, remédie à cela. Ce point distingue le libéralisme " moderne » à la fois du libéralisme classique et du libertarisme moderne ${ }^{19}$. Mais cette conception de

\footnotetext{
${ }^{18}$ On trouvait une remarque semblable chez Platon, à propos des femmes.

${ }^{19}$ N. Scott Arnold, Imposing Values, Oxford, 2009.
} 
l'égalité des chances laisse encore à la nature (le génome) et à la famille le soin d'influencer fortement les places dans l'échelle des revenus. Le principe de différence garantit que cela se fera au plus grand bénéfice possible des plus démunis, sans nier que d'autres puissent être incités par une espérance de gain plus grand à exercer leurs talents, ce qui introduira des inégalités contingentes inévitables. Mais la réciprocité dans les avantages mutuels et les incitations à la coopération plutôt qu'au conflit contribueront à ce que chacun, même le moins bien loti, soit en mesure de réaliser tout ou partie de son " plan de vie " et ce faisant, de se respecter lui-même en étant respecté par les autres coopérateurs.

$D^{\prime}$ autres conceptions lexicales sont possibles ${ }^{20}$, par exemple celle qui substituerait au PD l'utilitarisme (MaxUt), de préférence celui de la moyenne, au cas où la population augmente, car dans ce cas, comme le montre Rawls, avant Parfit ${ }^{21}$ selon moi, l'utilitarisme " total » exige le passage de $(2,2)$ à $(1,1,1,1,1)$ (car $5>4)$, alors que le bien-être des deux premiers diminue de moitié, quand l'utilitariste de la moyenne préfère $(2,2)$ (car $2>1$ ), ce qui doit plonger l'utilitariste dans un dilemme, comme l'avait compris Sidgwick. On peut aussi substituer au PD l'utilitarisme contraint par un minimum de survie. Rawls utilise plusieurs arguments contre de telles " conceptions mixtes », en particulier ceux de la réciprocité, de la "publicité » au

\footnotetext{
${ }^{20} \mathrm{TJ}, \S 49$.

${ }^{21}$ Reasons and Persons, Oxford, 1984.
} 
sens kantien, et de la difficulté qu'il y aurait à fixer un seuil minimal de revenu, lexicalement antérieur, contraignant MaxUt, sans le lier de quelque manière à une forme du PD. J'ai tenté d'y ajouter un argument sur la plus grande compacité des Deux principes par rapport au caractère ad hoc des conceptions mixtes. Ils expriment une même conception générale de la justice ${ }^{22}$, au lieu d'être la mise en ordre ad hoc de principes de nature différente.

Mais le principal argument de Rawls contre l'utilitarisme est centré sur la question de la personne humaine, ou du « soi » (self). Cet argument est partiellement au moins accessible dans la PO, que Rawls, fidèle au contractualisme rousseauiste et kantien, considère à la fois comme une méthode de choix et comme un outil moral, modélisant les exigences morales que l'on peut avoir concernant tout débat éthique bien conduit, par exemple l'impartialité et l'égalité des parties. Les décideurs soumis au VI sur leurs singularités, se savent néanmoins être des citoyens libres et égaux, et capables de de se donner chacun une conception rationnelle du «bien » (good), de ce qui vaut la peine d'être poursuivi comme un ensemble de fins dans la vie : c'est la seule défense de leur conception du «bien » (qu'elles ignorent) que les décideurs (artificiels) dans la PO vont rechercher en choisissant des principes capables de la protéger; mais les personnes réelles ont aussi un "sens de la justice », une " capacité » qui leur permet de comprendre et d'agir d'après des principes raisonnables

\footnotetext{
${ }^{22}$ Apologie, p. 131-2.
} 
de justice qui spécifient les termes équitables de leur coopération sociale. Ce sens n'est pas supposé motiver dans la PO, car c'est un sens hautement moral, mais il est en quelque sorte préfiguré formellement dans sa construction par l'égalité des parties, reconnue par toutes, et par l'égalité des restrictions informationnelles auxquelles elles sont soumises. On voit donc que, dès la PO, s'esquisse une conception complexe de la personne, qui s'épanouira, tout VI étant levé, dans la société bien ordonnée par les principes équitables de justice qui auront été choisis pour défendre la conception particulière du sens de la vie qu'entretient chacun (et que les "personnes artificielles» dans la PO ignorent par construction : mais elles, ou leurs représentants, savent qu'elles en ont une, et désirent pouvoir vivre selon elle).

Sans aucunement nier la pertinence des conséquences de telle ou telle conception, ce qui serait "délirant » (crazy), l'approche déontologique de Rawls, à l'opposé des approches "téléologiques " comme l'utilitarisme et le perfectionnisme, ne pose pas d'abord une caractérisation de ce qui a de la valeur, le " good", définissant alors le "right» (le moralement correct, le "Juste ") comme ce qui maximise ce " good » unique, mais il pose la priorité du Juste, dont la définition servira de filtre aux diverses conceptions du "good". Celles qui sont injustes ne seront pas prises en compte du tout. La personne rawlsienne est hiérarchisée : au-dessus de ses préférences 
pour les "biens premiers ${ }^{23}$, elle a des "fins ultimes ", qu'il est irrationnel de vouloir réduire à l'une d'entre elles en tant que « fin dominante ": le sujet "se défigure lui-même par esprit de système ${ }^{24}$. Autant l'intuitionnisme pluraliste est un mauvais candidat pour fournir une conception commune de la justice, autant les personnes réelles ne doivent pas défigurer leur diversité interne, en faisant tout dépendre de Dieu, ou du plaisir. Notre conception des fins de l'existence n'a pas besoin d'être gouvernée par une seule fin, et nous pouvons en avoir d'incommensurables. Nous sommes,

\footnotetext{
${ }^{23}$ Les biens sociaux que chacun est censé désirer " plutôt plus que moins ", dont les libertés, les responsabilités, la richesse et le revenu, et les bases sociales du respect de soi. Sans cette large classe de biens (objectifs), aucune théorie de la justice distributive ne serait possible. Mais il y a d'autres biens, dont certains plus "métaphysiques ", que chacun ignore et ne prend pas en compte dans la PO. Rawls ajoutera à sa liste des "primary goods", qui se substituent au bien-être ou à l'utilité, le loisir et même l'absence de douleur durable. II ajoute le loisir pour répondre à l'économiste Musgrave en 1974, qui accusait le PD de conduire à subventionner des personnes refusant de coopérer par leur travail, tels des moines ne désirant que prier. Ce point est le lieu du débat de haut niveau avec Philippe Van Parijs. Tout le monde veut plus ou moins de temps de loisir ; si des personnes choisissent d'y consacrer tout leur temps, la société selon Musgrave et Rawls n'a pas à leur fournir un revenu mesuré à l'aune du PD. Ce sont des "free riders", puisqu'ils ne peuvent pas souhaiter l'universalisation de leur comportement. (L'égoïsme du "resquilleur » est le type même de la maxime non universalisable au sens kantien : il ne faut surtout pas que tout le monde resquille, si je veux pouvoir le faire). Van Parijs insiste sur le fait que les effets de " resquillage » sont moins à craindre que ne sont à espérer les effets bénéfiques du revenu universel sur la formation, la culture et la recherche de nouvelles compétences, ce qu'il aurait peut-être pu appuyer sur ce que Rawls appelle le "principe aristotélicien ». Par ailleurs, dans le même ouvrage (Justice as Fairness), mais aussi dans LP, Rawls accepte (§ 13.3, note 7) l'idée du marxiste Peffer en ajoutant un principe lexicalement antérieur aux deux principes, celui d'un " minimum social ", permettant à tous les citoyens d'exercer leurs droits, et le suppose même plus essentiel, du point de vue constitutionnel, que le PD, alors que, en $\S 53.2(J F)$, il reprend l'exemple des surfeurs de Malibu, contra le revenu universel inconditionnel, pour justifier l'introduction du loisir dans l'index des biens premiers, et exprimer l'idée que tous les citoyens doivent "faire leur part du travail coopératif dans la société ". Le minimum social serait fixe et inférieur à ce que les surfeurs qui passent leur journée à surfer pourraient vouloir au titre du PD ? Ce point est délicat. Voir Van Parijs et Vanderborght, Le revenu de base inconditionnel, La Découverte, 2019.
}

${ }^{24} T$ J $\S 83$. 
individuellement, des "intuitionnistes " pluralistes, quant au bien. De plus, cet ensemble de fins, qui constituent l'essentiel de notre conception du "bien ", de la vie bonne, est changeant et révisable : les personnes ont une capacité rationnelle de changer de fins sans changer de personnalité au sens moral et juridique ${ }^{25}$. De plus, audessus de toutes leurs fins, elles ont donc les deux pouvoirs moraux. La personne rawlsienne est non atomique en ce sens qu'elle a une structure interne hiérarchique, mais aussi parce qu'elle n'est une vraie personne qu'au sein de pratiques et "d'unions sociales" (Humboldt) ${ }^{26}$. Nous sommes des personnes séparées, mais ouvertes à la coopération et essentiellement incomplètes. La personne est pour autrui source d'exigences légitimes (legitimate claims). Cette idée d'autrui comme (autre) source d'exigences légitimes est à mon sens exaltante et belle. Ne te considère pas seulement toi-même comme source d'exigences légitimes à l'égard d'autrui, mais considère toujours aussi et d'abord autrui comme source d'exigences légitimes pour toi! Parmi elles, son inviolabilité et son intégrité, qui seront donc protégées par le premier principe. Nous ne sommes pas loin de l'idée chère à Locke et aux libertariens de " propriété de soi ", même si Rawls n'emploie pas cette expression, ne risquant donc pas de tomber dans l'aporie selon laquelle la nue-propriété, ou jus abutendi, implique non seulement la possibilité de se suicider (ce qui n'est plus

\footnotetext{
${ }^{25}$ Rawls prend dans $L P$ l'exemple de Saül de Tarse, qui, avant le chemin de Damas, participait au martyre des chrétiens (Étienne).

${ }^{26} T J, \S 79$.
} 
un crime), mais aussi celle de (se) vendre (esclavage volontaire) $)^{27}$. Le "juste » interdit cela, et ne prend pas du tout en compte les éventuelles conceptions du « good » qui le tolèrent.

Au rebours de cette conception complexe de la personne, du sujet, la conception utilitariste de la personne apparaît comme presque inexistante.

1) Les individus sont bien égaux, mais ils ne sont pas entourés d'une armure de droits inviolables, constituant pour les autres et pour les décideurs sociaux autant d'interdits ${ }^{28}$. Les droits ne peuvent qu'être, à la rigueur, dérivés à partir de calculs (problématiques) montrant que leur respect augmente I'utilité globale. (Ils sont plus admissibles au sein de "l'utilitarisme de la règle " que de celui " de l'acte", lequel est cependant le plus obvie.)

2) L'utilitariste applique à la collectivité des raisonnements économiques adaptés à l'individu, producteur ou consommateur. Ce sophisme « collectiviste ${ }^{29}$ fait qu'il « ne prend pas au sérieux la distinction des personnes ${ }^{30}$. Rawls parle aussi de " séparation des personnes ». J'ai été heureux

\footnotetext{
${ }^{27}$ II y a quelque chose de séduisant, surtout si l'on pense au harcèlement sexuel, dans l'idée selon laquelle il faut voir sur le front de chaque personne quelque chose comme: " propriété privée : ne pas toucher ou utiliser sans mon consentement exprès ". L'intégrité de la personne joue ce rôle dans le système rawlsien.

${ }^{28} T J, \S 1$, par. 1 ; ab initio.

${ }^{29}$ Opposé à l'individualisme méthodologique des Autrichiens, en particulier Popper.

${ }^{30} T J$, §5, dernière phrase, citée un nombre incalculable de fois.
} 
de trouver l'expression même de "personnes séparées " sous la plume de Descartes écrivant à Élisabeth ${ }^{31}$.

3) L'utilitarisme pense les individus comme substituables, et autorise à « sacrifier » les intérêts de certains si cela est plus que compensé par l'amélioration du bien-être agrégé d'autres individus du collectif concerné. On doit passer de $(2,3)$ à $(1,5)$, puisque la somme des utilités est supérieure dans le second état. Le modèle théologique de cette pente pourrait être à mon sens la théodicée leibnizienne : le viol de Lucrèce est plus que compensé par ceci que "le crime de Sextus sert à de grandes choses ${ }^{32}$. Ce point fait système avec le premier. Lucrèce n'est pas garantie dans son inviolabilité, compensable par une somme de biens plus grande.

4) Les individus ne comptent pas en tant que tels, mais seulement en tant que "conteneurs" de satisfactions. Un conteneur n'a que très peu de valeur. Ce qui vaut, c'est ce

\footnotetext{
${ }^{31}$ Apologie, p. 119.

${ }^{32}$ Théodicée, Troisième Partie, dernière page. Leibniz critique par ailleurs Malebranche et sa théorie du choix divin du meilleur des mondes, compte tenu du fait que les lois en doivent être simples, et affirme au rebours que Dieu, pour ainsi dire, maximise doublement (voir Elster, Leibniz, 1975, ch. V). Sans commettre cette erreur mathématique, dénoncée par Rawls lui-même ( $T J, \S 17)$, le créateur du calcul des variations aurait pu simplement signifier par-là que les singularités que sont les maxima présentent aussi des propriétés qualitatives notables (ex: dérivée nulle). Il me vient à l'idée que cela pourrait provenir de ceci que la position malebranchienne correspond à un "pluralisme intuitionniste ", sans qu'en Dieu apparaisse une raison (morale) de choisir telle ou telle pondération de perfection et de simplicité. Ajoutons, puisqu'il est question de la rétribution d'un crime, qu'il est fort dommage que Rawls n'ait pas élaboré une théorie pénale pour les "sociétés non idéales ». II renvoie à Hart.
} 
qui le remplit, en l'occurrence, des plaisirs, ou, plus généralement, des satisfactions. La personne utilitariste est une pseudo-personne, "a bare person", une personne indéterminée, sans caractère ni volonté propre. Une machine désirante...

5) L'utilitarisme ne peut opérer de distinction entre les devoirs et les actes "surérogatoires": moralement permis, moralement non obligatoires, et moralement louables. C'est une morale complète, ce que ne propose pas Rawls, mais une morale altruiste hyper-exigeante, de "saints et de héros ». Une société bien ordonnée ne doit pas avoir des principes qui pourraient gouverner "même un peuple de démons " (Kant), mais elle peut être composée de personnes justes, se respectant, tout en ne passant pas leur temps à se sacrifier. Elle est « une entreprise commune visant l'avantage mutuel ", de telle manière que l'amélioration de l'un s'accompagne toujours d'une amélioration de l'autre (réciprocité). La réciprocité est plus exigeante que l'égoïsme, bien sûr, mais moins que l'héroïsme. Nous pouvons admirer les héros, car ils font plus que leurs simples devoirs.

Rien ne dit que les utilitaristes ne puissent apporter de réponses à ces objections philosophiques, mais nous les supposerons valides, à I'instar de Nozick et Sandel. 
L'argument que j'appelle "Tu Quoque ! » consiste à admettre ce qui précède, en particulier la thèse de la séparation des personnes, et à tenter de montrer que le PD rawlsien revient à détruire l'idée de personne (séparée) à laquelle son auteur est à juste titre tant attaché. Nozick consacre cinquante pages de son ouvrage à la $T J^{33}$, et ses critiques les plus radicales sont concentrées dans les dernières pages, intitulées "Collective Assets ». Cette expression (" atouts communs/collectifs", "actifs communs", voire "ressources communes ») est utilisée dans la section 17 de la $T J$ : "La tendance vers l'égalité. " Avant que de défendre le choix du PD (en tant que dernier principe) contre le perfectionnisme et l'utilitarisme dans la $\mathrm{PO}$, où les raisonnements des agents, soumis à des contraintes argumentatives morales, ne sont pas, quant à eux, motivés par la moralité, mais par le seul intérêt rationnel, Rawls se propose de le défendre d'abord par des arguments directement éthiques, hors PO. Le PD nous amène, dit-il, à changer notre point de vue sur le social, en s'écartant des valeurs technocratiques, mais aussi de l'idée que les mérites naturels seraient une base non arbitraire de distribution des biens premiers, les objets de la justice distributive. En fait, c'est selon lui un truisme moral que nous ne "méritons" pas plus nos qualités innées que nous ne méritons d'être né(e)s dans telle ou telle milieu socio-culturel: ce ne sont que des faits non moraux, « arbitraires d'un point de vue moral ». La moralité, c'est ce que l'on

\footnotetext{
${ }^{33}$ Anarchie, État et Utopie, ch. 7, section II.
} 
en fait. Ne peut-on pas au moins atténuer l'effet de ces facteurs moralement arbitraires ? Le PD nous permet d'envisager autrement la distribution " différentielle » des talents (un tel est plus rapide, une telle plus créatrice, une autre a plus de voix), à savoir comme si c'était un "collective asset ». Et Nozick de s'insurger: faire de l'ensemble de nos talents une propriété publique, c'est pire que du communisme! Lui-même, comme Rawls, se réclame de la plus notable formulation de l'impératif catégorique : "Traite l'humanité, aussi bien dans ta personne que dans la personne de tout autre, toujours en même temps comme fin, jamais simplement comme moyen ! ${ }^{34}$ Or, si nos qualités, nos dons, ne nous appartiennent plus, à cause du PD qui les socialiserait, ils sont traités seulement comme des moyens par la société rawlsienne, et "nous" en sommes détachés. Seul en nous un soi « purifié ", sans qualités, serait à traiter d'abord comme une fin en soi $?^{35}$ Incohérence flagrante, ou fantaisie métaphysique, d'autant que l’on ne peut plus « prendre au sérieux la distinction des personnes ", puisque ce qui les distingue, leurs dotations ou talents, sont collectivisés. L’horreur! Une société pire

\footnotetext{
${ }^{34}$ Fondation de la Métaphysique des Mœurs, Section II, GF. On la paraphrase souvent en omettant le terme "humanité " et le fait qu'elle s'applique à la personne elle-même (suicide, onanisme) autant qu'à l'autre.

35 Cette critique peut faire écho à certains scolastiques, tel Dietrich de Freiberg, qui reprochaient à l'interprétation thomiste de la transsubstantiation de séparer une substance "pure ", ayant seule l'aséité, de ses accidents. Sans prendre parti dans cette querelle métaphysique, je soutiens juste qu'elle ne concerne pas le sujet rawlsien, toujours empirique et plein de qualités qui sont autant d'aspects de soi qui appellent une complémentarité avec les autres, comme, si j'ose dire, l'amour selon Aristophane dans le Banquet. Rawls critique finement l'utilitarisme classique pour sa conception de l’amour/bienveillance fusionnel, dans la $§ 30$.
} 
que celle de 1984 ou que celle de Harrison Bergeron $^{36}$ se profile, qui ferait de notre décision libre de ne pas faire fructifier tel ou tel talent la « dilapidation d'un bien public, un délit ! ».

L'erreur de Nozick, que dénoncera Rawls discrètement dès $1974^{37}$, réside en ceci que ce n'est pas l'ensemble des talents qui est « vu comme » un actif collectif, mais leur distribution, autrement dit leurs différences. Cette distribution inégale peut être saisie collectivement comme une saine base pour la coopération à travers la division du travail, comme l'avait vu Adam Smith. Regarde toujours l'autre non pas d'abord comme un adversaire, mais comme un partenaire d'une coopération possible $!^{38}$ Tous nos talents en ce sens sont des sources de complémentarités possibles. Ce sont bien les distinctions entre des personnes séparées qui leur permettent et les incitent à coopérer. Comme les deux morceaux d'un sumbolon, nos complémentarités peuvent s'emboîter. Le PD s'empare en quelque

\footnotetext{
${ }^{36}$ Nouvelle de Kurt Vonnegut, souvent citée dans les débats américains sur l'égalité. Les personnes trop belles ou trop intelligentes y sont munies de masques ou de radios de handicap mental. On la trouve en appendice du très sérieux recueil Equality, Selected Readings, ed. Louis Pojman \& R. Westmoreland, Oxford University Press, 1997.

37 " A Reply to Alexander and Musgrave “, Collected Papers, Harvard, 1999, p. 246, note 6.

${ }^{38}$ La concurrence honnête est elle aussi la bienvenue, pour tous les libéraux, hostiles aux monopoles et favorables à ce que les marchés soient au moins "contestables ". La complémentarité s'exerce surtout dans les entreprises, ou entre des entreprises participant à une même chaîne de production. La règle d'or en est : tous les coopérateurs doivent profiter positivement de la coopération, sous peine d'être un "sucker ", un "pigeon ", comme l'on dit en théorie des jeux de celui qui coopère naïvement, alors que l'autre fait défection (free rider). II me semble que même le marché concurrentiel, bien entendu conflictuel, a quelque chose d'une coopération, fixée pour toujours dans notre imaginaire par la "main invisible " smithienne, bien sûr insuffisante. Une équipe de rugby doit être intensément coopérative, mais les deux équipes rivales "coopèrent " à un autre niveau, pour produire « du beau jeu ».
} 
sorte de ce fait, la différenciation des talents, pour inciter les personnes à les faire fructifier par la coopération, de telle manière que chacun en profite, y compris les moins chanceux, dont le sort est maximisé, étant donné les inévitables inégalités induites par les incitations et le hasard. II manifeste ceci que la société tout entière est une "entreprise de coopération pour l'avantage mutuel», et contribue donc " aux liens de l'amitié civique ${ }^{39}$. II permet de voir ainsi nos différences de talents non comme des motifs d'envie, passion anti-sociale, mais comme des atouts pour tous. La société juste est à comparer avec un orchestre, où chacun joue sa partition, plus ou moins importante, mais où chacun compte, et peut être respecté et se respecter lui-même ou elle-même en tant que membre actif de l'œuvre commune ${ }^{40}$. Exit, le contresens de Nozick. Nos talents ne sont pas séparés de nous, et cela est garanti par "l'intégrité de la personne » dûment mentionnée dans le premier principe $^{41}$. Ils nous appartiennent, mais comme ils ne peuvent fructifier que grâce à la coopération sociale, chacun peut comprendre qu'une partie de leurs fruits puisse servir à tous.

Un argument de Nozick, cependant, me paraît exact. Le PD, qui n'autorise que des augmentations d'inégalité qui profitent positivement à tous, ne saurait accepter la progression parétienne

\footnotetext{
${ }^{39} T J$, § 1. Phrase typiquement aristotélicienne et "républicaine ».

$40 J F, \S 21.3$.

${ }^{41}$ Il faut admettre que ceci que la garantie que nos talents sont les nôtres est impliquée par "l'intégrité de la personne " ne serait peut-être pas devenue évident si Nozick n'avait pas amené Rawls à préciser ce point crucial.
} 
(seulement faible) entre $(5,8)$ et $(5,10)$, si l'ensemble faisable se réduit à ces deux seuls états, puisque le plus mal loti, quel qu'il soit, $n^{\prime}$ en profite pas. Nozick reconnaît que Rawls ${ }^{42}$ semble accepter la suggestion faite dès 1970 par Amartya Sen d'adopter un Maximin lexical, plus tard appelé "Leximin", lequel consiste à réitérer le Maximin sur chaque place; on choisit d'abord les états qui maximisent la situation du plus mal loti, puis, dans le sous-ensemble faisable qui demeure, on départage les ex aequo en maximisant les " seconds plus mal lotis », et, à la fin, s'il demeure $(5,8)$ et $(5,10)$, le Leximin choisit le second (comme le ferait la règle MaxiMax: maximiser la situation de celui qui en a déjà le plus), puisque celui qui occupe la place de droite est le tout dernier " mal loti ». Mais Nozick note à juste titre quelque "unclarity " dans la position de Rawls eu égard au Leximin, arguant par ailleurs, comme le retiendra le marxiste G. A. Cohen, que ce principe n'incorpore pas la présomption en faveur de l'égalité que Rawls met en avant en faveur du PD. II nous faut tenter de lever ces graves ambiguïtés. Revenons à l'étude des différentes règles de justice.

L'égalitarisme radical, que l'on caractérisera comme la mise en pratique de la règle de choix social MinIN (" Minimisez l'inégalité ! »), se heurte à l'idée qu'elle conduit automatiquement à préférer l'état $(1,1)$ à l'état $(3,5)$, où toutes les personnes sont pourtant mieux loties, mais où l'inégalité passe de 0 à 2 . Empêcher une telle transition au

\footnotetext{
${ }^{42} T J, \S 13$, in fine.
} 
nom de Minln, par esprit de système, c'est donner le flanc à ladite "Levelling down objection", discutée par Parfit et Temkin ${ }^{43}$, ou " objection du nivellement par le bas ». L'égalitariste radical préfère une situation où tout le monde est dans la (même) misère à une situation où tout le monde va nettement mieux, mais où il $\mathrm{y}$ a de l'inégalité. Minln (en tant que principe moniste) récuse toute progression parétienne (progression approuvée par au moins l'un des agents, et désapprouvée par aucun), sauf celles qui maintiennent la même inégalité : de $(1,1)$ à $(2,2)(\ln =0)$, ou, dans un autre ensemble faisable, de $(1,2)$ à $(2,3)(\ln =1)$ par exemple. En toute rigueur, elle est en quelque sorte indifférente entre ces états, dans chaque paire, (tout en préférant les deux premiers aux deux autres), mais si on lui adjoint comme règle lexicalement secondaire, pour sortir des ex æquo, la règle utilitariste MaxUt (ou le Maximin, confondu avec le PD), on obtient certaines progressions parétiennes fortes, à condition qu'elles ne modifient pas l'inégalité de départ, idéalement nulle. En notant « + " l'opération (non commutative) consistant à ajouter une règle lexicalement secondaire à la première, on peut, dans le cas d'états composés de deux individus ou deux classes, éliminer toute " courbe d'indifférence ». Ainsi, la règle complexe Minln + MaxUt conduit à : $(2,2)>(1,1)$, alors que Minln seule les situe sur une même courbe (demi droite) d'indifférence, la bissectrice Nord-Est de l'égalité parfaite. Par ailleurs, MinIn préfère évidemment les états en

\footnotetext{
${ }^{43} \mathrm{Cf}$. The Ideal of Equality, ed. M. Clayton \& A. Williams, Palgrave Macmillan, 2002.
} 
question, où l'inégalité est nulle, s'ils sont réalisables, mais continue sinon à préférer $(1,2)$ à $(2,5)$, alors que chacun profite du passage du premier état au second (progression parétienne forte), ce qui est alors requis au contraire par Maximin, la règle qui enjoint de maximiser la position de ceux qui en ont le moins. L'utilitarisme conduit quant à lui à préférer $(1,10)$ à $(5,5)$ au motif que $11>10$, ce qui est contre-intuitif. Le perfectionnisme ne peut être représenté par de tels chiffres, mais il est a priori peu compatible avec l'idéal démocratique, que ce soit dans sa version aristotélicienne idéale (« le vertueux »), ou dans sa version nietzschéenne (le "surhomme »). Quant au libertarisme, il nous dit que si un processus de marché concurrentiel libre a conduit par des échanges justes à l'état Pareto optimal $(3,10)$, si donc des états tels que $(4,10)$ ou $(3,11)$ ne sont pas faisables, il n'y a strictement plus rien à faire pour obtenir un résultat plus juste: ce serait voler celui qui a 10 pour le "redistribuer» au plus malchanceux, ou moins doué. Le minarchisme de Nozick, ou libertarisme non anarchiste (État minimal), est donc a priori compatible avec n'importe quel niveau d'inégalité in fine (comme l'utilitarisme pur, mais pas du tout pour les mêmes raisons). N'exigeant pas de transformations non parétiennes («qui ne plaisent pas à tous »), il s'oppose à presque toutes les autres théories de la justice, y compris l'utilitarisme, lesquelles, ne considérant que les états, sont conduites par leur logique à autoriser/exiger certaines transformations non parétiennes, 
autrement dit qui lèsent au moins une partie. MaxUt préfère ainsi $(1,10)$ à $(2,8)$, alors que ce sont des états Pareto-incomparables (il y a un veto mis à tout passage de l'un à l'autre), et que le premier individu, le plus mal loti, "y perd » $(1<2)$. Minln quant à elle, va préférer en rester à $(2,2)$ plutôt qu'à passer à $(3,5)$, on l'a dit, transformation qui correspond pourtant à une progression parétienne forte (unanimement approuvée, positivement, sans veto ni abstention). La règle Maximin, formellement analogue à son homonyme hyper-prudente en théorie de la décision ${ }^{44}$, conduit à accepter toutes les progressions parétiennes fortes (lesquelles sont $a$ fortiori faibles) ${ }^{45}$, telles que $(2,3)->(3,5)$, et demeure « indifférente » entre tous les états liés seulement par des progressions parétiennes seulement faibles, telles que $(2,3) \rightarrow(2,5)$ : ses courbes d'indifférence sont celles des «biens parfaitement complémentaires ", en $L$, comme celles que trace Rawls pour

\footnotetext{
${ }^{44}$ Et utilisée, au grand dam d'Harsanyi, et de bien des économistes, par Rawls dans la PO, pour justifier la préférence unanime en faveur des deux principes (mais pas du PD en particulier, affirme JasF). Ne sachant rien de la probabilité que je sois dans telle ou telle situation, je vais me garantir, pour moi et ma famille, un minimum inviolable de biens premiers, en particuliers les droits, "comme si mon pire ennemi avait choisi ma place »: même si un Malin Génie, dirais-je en parlant comme Descartes, devait me situer dans la société, il ne pourrait pas ne pas faire que j'existe comme personne, entourée et protégée par des droits. Un « cogito " rawlsien...

${ }^{45}$ Me fiant à la dernière minute à une définition fautive donnée par le théoricien des jeux Ken Binmore (Playing Fair, MIT Press, 1994), j'ai malheureusement, dans le ch. V de l'Apologie, remplacé "faible » par "forte » et réciproquement. Le lecteur, que je prie humblement de m'excuser, doit rétablir la bonne terminologie (uniquement dans les expressions du type "la progression parétienne faible/forte »). Les définitions de G. A. Cohen sont correctes.
} 
commenter son principe de différence ${ }^{46}$. Il y a là une ambiguïté dans la $T J$, que son auteur n'a semble-t-il jamais perçue. Comme l'a bien vu Parfit ${ }^{47}$, entre $I=(2,3)$ et $I I=(2,4)$, tout se passe comme si Rawls aurait hésité entre trois positions logiquement possibles : soit I > II, soit II > I, soit I et II sont indifférents (et donc sur une même demidroite d'indifférence des courbes en $L$ de ses figures 5 et 6 dans la $\S$ 13 de la $T J$ ). Comme G. A. Cohen, Parfit assimile la deuxième position au dit Leximin, inventé donc en 1970 par Amartya Sen pour éviter au principe de différence (déjà publié par Rawls, depuis 1958) d'avoir à être indifférent entre $(1,2)$ et $(1,20)$, et assure que c'est là l'authentique PD de Rawls. Là contre, j'ai argüé que le Leximin, qui réitère le Maximin à chaque position, finit en dernier lieu par préférer, disons, $(3,10)$ à $(3,4)$, comme le Maximax, alors que Rawls énonce toujours le PD de telle sorte qu'il en ressorte que tous les individus (ou classes) profitent positivement de toute augmentation d'inégalité. Le PD n'est donc pas « indifférent " : il préfère $(3,4)$ à $(3$, 5), si le choix se réduit à ces deux états, car l'augmentation de richesse du second (celui qui est le plus riche) n'est accompagnée d'aucune amélioration de la situation du premier, et n'est donc pas justifiable (pas réciproque). Seule la règle Maximin a des courbes d'indifférence, mais ni le Leximin ni le PD stricto sensu n'en ont. Je propose de construire le PD, dans le cas de deux classes, comme la

\footnotetext{
${ }^{46} T J$, § 13, fig. 6 ; Apologie, fig. p. 197-99.

47 "Equality or Priority", in The Ideal of Equality, op. cit., ch. 5 (Appendix: Rawls's View.)
} 
« somme » lexicale de Maximin + MinIN, alors que le Leximin revient à appliquer Maximin + Maximax ${ }^{48}$.

Il est obvie pourtant que, dans la PO, tout décideur rationnel va préférer $(3,5)$ : ne connaissant pas sa position, il appert que s'il a la première, cela l'indiffère, et que s'il occupe la seconde, il préfère le second état, car $5>4$; en fin de compte, il préfère donc $(3,5)$. Il faut me semble-t-il imaginer, comme Rawls le fait à la fin de la $\S 17$, que si la transformation seulement faible en question est possible, alors il est assurément (surely) possible de passer de $(3,5)$ à $(3+n, 5-n)$, élargissement de l'ensemble faisable que l'existence de l'État, connue dans la PO, en tant qu'institution de base de la société, rend possible : qui dit État dit fisc, et le fisc peut être utilisé à des fins redistributives; on ne voit pas au nom de quoi l'on pourrait interdire ce savoir dans la PO, où les décideurs ne sont pas informés de ceci que «seules les fonctions régaliennes de l'État peuvent être financées par l'impôt ", ce qui à l'évidence est faux, ou normatif (minarchiste); or, Rawls précise bien qu'on ne leur donne pas d'informations fausses et qu'ils ou elles n'ont pas " déjà " de théorie normative. Or, dans ce cas, si $\mathrm{n}$ toutefois n'est pas si grand que sa soustraction à la somme obtenue par «le mieux loti » ne le " désincite » pas à innover, à prendre des risques, la règle Maximin enjoint de choisir $(3+n, 5-n)$, en partant de $(3,4)$, ce qui est une

\footnotetext{
${ }^{48}$ De ce fait, le Leximin n'est pas égalitariste, comme le souligne Cohen, après Nozick ; de ceci qu'il le considère néanmoins comme la version " canonique " du PD, il peut en tirer la conclusion que ce dernier principe n'est pas un principe de justice. Ce que je récuse donc.
} 
progression parétienne forte. II n'y a dans ce raisonnement que des éléments de connaissance générale, accessibles en principe sous le VI, comme le sont les "circonstances de la justice » humiennes. Rawls a pu laisser croire (ou croire) que la figure 6 avec les courbes en $L$ représentait le PD, et que celui-ci était donc indifférent entre (2, 3 ) et $(2,10)$, mais ce uniquement parce que la courbe de production OP qu'il trace incarne ce qu'il appelle la "close-knitness » de la société $^{49}$ : tout changement dans la position de l'une des (deux) parties est accompagné d'un changement dans la position de l'autre. La « close-knitness» (« couplage étroit ») exprime la " soudure » des citoyens. Le sort de l'un réagit au sort de l'autre. Tant que la situation du plus mal loti monte, le PD enjoint, à partir de $\mathrm{O}$, de monter jusqu'au sommet unique $D$, où la dérivée s'annule. $D$ n'est suivi d'aucun segment plat. Si l'on est dans une société telle que l'augmentation des inégalités profitent aux plus démunis, on continue de profiter de cette augmentation. Toute montée à gauche de $D$ est une progression parétienne forte, et $D$ est le premier état Pareto optimal au sens faible (et fort si D est unique, grâce à la "close-knitness ») obtenu en partant de $\mathrm{O}$, et le plus proche de la bissectrice de l'égalité : ce que l’on peut appeler le "résultat de Rawls ", pour ne pas se risquer à parler de "théorème ". Après le point $D$, unique (close-knitness), tous les états sont des optima de Pareto au sens fort (et donc a fortiori faible) et, au contraire, le PD

${ }^{49}$ TJ, § 13. Voir Apologie, p. 197-201. 
enjoindra de remonter vers $D$, en abaissant la richesse des plus aisés, en la transférant aux plus démunis (par tous moyens pacifiques), ce qui est une transformation non parétienne entre états Pareto optimaux au sens fort (incomparables par les seuls concepts parétiens). Le PD, selon le type de société dans laquelle on se trouve (I'une, avant $D$, où l'on peut encore augmenter les incitations, l'autre, après $D$, où celles-ci sont gaspillées, du point de vue de la justice, puisqu'elles ne servent plus à améliorer aussi les attentes sur toute une vie des plus faibles, mais servent uniquement à enrichir les plus riches), enjoint donc soit des transformations non parétiennes ${ }^{50}$, si l'on est à droite de $\mathrm{D}$ (diminuant la part des plus riches), soit des progressions parétiennes fortes, et donc faibles aussi, interdisant selon moi ce qu'autorise/exige le Leximin (comme d'ailleurs $\mathrm{MaxUt})^{51}$, à savoir les progressions seulement faibles, où les pauvres stagnent, tout en contemplant l'ascension des plus riches, de plus en plus haut, de plus en plus loin d'eux. On note que le point $D$ n'est pas le point où le $\mathrm{PIB}^{52}$ est maximal. Ce but politique (puissance nationale) n'est pas en soi une finalité juste. Par ailleurs, Rawls accepte l'idée de Mill d'un état maximal de production, où la croissance sera nulle, stationnaire (et la croissance démographique

\footnotetext{
${ }^{50}$ Ce doit être le cas aux USA, pensait à coup sûr le philosophe de Harvard, sévère sur son pays.

${ }^{51}$ Comme l'utilité de personne ne baisse et que celle d'au moins un individu augmente, l'utilité totale augmente, et la conséquence est bonne.

${ }^{52} \mathrm{Ou}$ sa version utilitariste, le « bonheur intérieur brut », au calcul encore plus incertain.
} 
non positive $)^{53}$. On pourra toujours cependant tenter de passer à des courbes OP dont le point $\mathrm{D}$ soit plus proche de la bissectrice de l'égalité, sur une même demi-droite d'indifférence Maximin. (En continuant à faire croître seulement le savoir, et en gardant le loisir comme bien premier, on pourrait non pas décroître, mais diminuer les externalités négatives de nos activités sur la nature, et augmenter le temps de loisir.)

La plus grosse difficulté me paraît être que rien ne vient nous aider à déterminer quelque chose comme le seuil maximal d'imposition où les riches entrepreneurs ou les jeunes innovateurs talentueux commencent à être "désincités ", ce qui serait mauvais pour les plus mal lotis. Une seconde difficulté vient de la concurrence fiscale, que Rawls n'évoque pas : si une société rawlsienne JR est en concurrence fiscale avec une nation libertarienne RN, sans impôts redistributifs, les talents entrepreneuriaux peuvent être tentés par l'exil en RN, ce qui est mauvais pour les plus mal lotis de JR. Le PD intégré comme un principe de justice par la plupart des citoyens de JR peut-il alors les inciter à baisser la forte pression fiscale sur les plus aisés, pour empêcher l'exil fiscal? (Cohen en déduirait que, décidément, les riches ne peuvent pas être moraux.) Une troisième difficulté survient si l'on admet que la partition de la société en «riches» et "pauvres» est insuffisante. Rawls imagine alors

\footnotetext{
${ }^{53} J F, \S 18.3$
} 
I'hypothèse de la "connexion en chaine ${ }^{54}$ : si les plus riches et les plus pauvres s'enrichissent (les plus riches plus vite que les plus pauvres), avant $D$, alors, les classes intermédiaires s'enrichissent aussi ; tout le monde est encordé, à ceci près que la corde est extensible : l'inégalité augmente jusqu'à $D$. Ce point est important, et suggère à mes yeux que le $P D$ est en principe fortement individualiste : tous les citoyens voient leur sort s'améliorer quelque peu, entre $O$ et D. Parler de "classes" ne serait qu'une approximation. Car il est bien connu par les théoriciens des inégalités que les différentes partitions que l'on peut faire d'une société donneront des indications différentes : si les plus riches sont ceux qui constituent le décile le plus élevé, le résultat pourra montrer une moindre augmentation d'inégalité que si le centile, voire le millième le plus riche est pris en compte (les milliardaires). Si l'on veut simplifier, comme le fait Rawls, en prenant deux classes (voire trois), on peut comme lui proposer d'intégrer aux "plus mal lotis " ceux dont le revenu tourne autour de celui des travailleurs non qualifiés, ou, autre mesure, ceux dont le revenu est inférieur à la moitié du revenu médian ${ }^{55}$. Mais si l'on veut complexifier la situation pour la rendre plus réaliste, il vient que plusieurs partitions sont possibles, et que le PD ne saurait plus être caractérisé par la simple "somme " lexicale de deux règles. II faut sans doute s'intéresser aux écarts à la

\footnotetext{
${ }^{54}$ Apologie, fig. p. 217.

${ }^{55} \mathrm{TJ}, \S 16$.
} 
moyenne, à la variance, sans aller peut-être jusqu'à appliquer l'indice de Gini. Sur ce dernier, Rawls ne s'étendit jamais : "Les principes de justice n'impliquent pas que la distribution effective se conforme à tout moment à quelque structure observable (disons, l'égalité), ou que le degré d'inégalité tombe dans un certain domaine, par exemple de valeurs du coefficient de Gini. " ${ }^{56}$

Peut-être que si le PD appliqué à plus de deux classes, malgré la contrainte de "connexion en chaîne ", demeure indéterminé, pourrait-on se contenter du nouveau critère proposé par l'économiste chilien José Gabriel Palme, qui ne prend en compte que les $10 \%$ les plus riches et les $40 \%$ les plus pauvres. Quoi qu'il en soit, je n'ai pas la possibilité d'aller plus loin, et je laisse cette éventuelle tâche aux économistes, bien plus compétents que moi en la matière. Le PD est avant tout régulateur, et se trouve, en tant que principe de justice, jouer un rôle fonctionnel à l'arrière-plan des politiques fiscales ou encore en matière d'héritage, lesquelles doivent le prendre en compte comme contrainte régulatrice, mais il n'a pas la capacité de déterminer la part de chacun dans des transactions particulières. La croissance, jusqu'à ce qu'elle atteigne son niveau maximal "stationnaire ", doit profiter à tous, et les inégalités ne doivent pas dépasser ce qui est nécessaire pour renforcer la motivation de ceux d'entre les citoyens qui choisissent de faire fructifier leurs talents entrepreneuriaux ou d'innovateurs, par

\footnotetext{
${ }^{56} L P, \mathrm{VII}, \S 9$ (avec un renvoi à Sen).
} 
exemple en faisant des études difficiles et coûteuses. La fixation de ce point de rebroussement, après lequel l'augmentation des inégalités ne profite plus aux plus démunis ne semble pas empiriquement possible.

Cela dit, Rawls aurait pu se contenter de rappeler et de raffiner les «fonctions musgraviennes » de l'État ${ }^{57}$, et de situer le mode de production le plus apte à réaliser quelque peu les ambitions des deux principes dans la tradition keynéso-béveridgienne, si l'on peut agglomérer ces deux perspectives différentes, ou dans la tradition sociale-démocrate européenne. Autrement dit, justifier un WelfareState (État-Providence) généreux, capable de réguler le marché, de fournir des " biens publics " (non rivaux ${ }^{58}$ et non " excluables ${ }^{59}$ ), de déterminer comment internaliser les externalités négatives (pollution...), de fournir des biens sociaux tels que l'éducation et la santé publiques, et de financer des politiques d'assurance et d'assistance publiques destinées à se substituer largement à la

\footnotetext{
${ }^{57} T J, \S 43$.

${ }^{58}$ Leur offre ne devient pas plus petite en étant consommée.

59 Disponible pour tout le monde (sur une aire donnée) : il est pour le moins difficile d'en faire payer l'accès. La défense nationale et la paix civile sont les deux biens publics essentiels qui justifient l'État hobbesien (Lv, ch. 13 et 17). Aucun anarchiste, ni Kropotkine ni Rothbard, n'a à mon sens expliqué comment ces biens (négation de "maux publics ") pourraient être fournis sans aucune autorité centrale monopolisant l'usage légitime de la coercition (Weber). Dans des sociétés plus grandes que les " tribus " paléolithiques (150 personnes ?) et plus complexes (division du travail, villes). Marx pensait que la complexité disparaîtrait avec le communisme, où tout serait, comme chez Robinson, "transparent et simple": tragique erreur. Dans le débat sur le « calcul socialiste ", c'est von Mises qui avait raison (cf. N. Scott Arnold, Marx's Radical Critique of Capitalist Society, Oxford University Press, 1990). Mais Arnold critique aussi finement le "socialisme de marché ", qui avait les faveurs de Rawls.
} 
souhaitable charité privée, de telle manière que chaque citoyen puisse exercer ses « deux pouvoirs moraux » et profiter du respect de lui-même. Or, contre toute attente, Rawls affirme que le capitalisme, même assorti d'un tel État social, ne saurait réaliser les deux principes.

Récapitulons l'ensemble des modes de production dont parle (ou aurait pu parler) Rawls.

$1^{\circ}$ Le communisme de Marx. Dans sa "seconde phase ${ }^{60}$, il se caractérise par l'absence de monnaie, d'État, de propriété privée et de marché. Les « entraves » à la croissance des forces productives dues au capitalisme ayant été détruites par la révolution prolétarienne, et une fois dépassée la «première phase », encore dépendante de la différence de productivité des travailleurs, et donc du Droit, " toujours inégalitaire ", l'abondance règne, le travail est réduit et toujours intéressant (non aliéné), chacun ne travaillant que selon ses capacités, mais de telle manière que les travailleurs n'aient plus à échanger le produit de leur travail, mais juste à les mettre à disposition des besoins de tous. Utopie irréaliste (comme l'anarchisme collectiviste, dont il est proche). La belle vertu de justice, remarque Rawls ${ }^{61}$, n'y a plus de sens : cette utopie se situe au-delà des « circonstances de la justice » au sens de Hume, et « audelà de la justice » (beyond justice).

\footnotetext{
${ }^{60}$ Critique du Programme de Gotha (1875).

${ }^{61}$ Lectures on the History of Political Philosophy, Oxford University Press, 2007, Lectures on Marx III.
} 
$2^{\circ}$ Le communisme "réel " (depuis 1917), où l'abondance ne règne certes pas, mais où, le marché ayant été supprimé, l'économie est dirigée centralement par l'État, "planifiée ", y compris les prix relatifs. Une catastrophe, comme les Autrichiens (Böhm-Bawerk, von Mises, Hayek) l'avaient correctement prédit. Système à parti unique, totalement incompatible avec la protection des libertés au sens du Premier principe. Et système à la fois inefficace (sauf peut-être du point de vue militaire) et injuste (anti-libéral et produisant de fait une nouvelle oligarchie cachée, la bureaucratie d'État communiste) ${ }^{62}$.

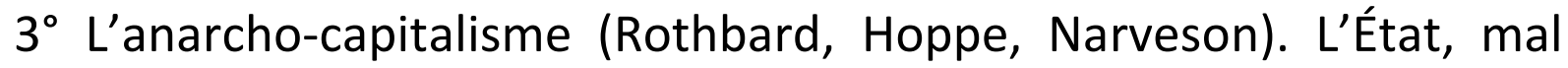
non nécessaire, ayant disparu, seuls demeurent les individus pleinement propriétaires de leur personne et de leurs biens, échangeant librement leurs produits sur le marché non entravé et non régulé. Les inégalités en sont le produit naturel, et les pauvres peuvent être soulagés de leur misère par la charité volontaire seulement. II n'y a plus d'impôt. Utopie irréaliste, dangereuse, injuste, refusée par les libéraux classiques (Locke, Smith, Mill, Hayek, Friedman...), et dont Rawls ne parle pas.

$4^{\circ}$ Le libéralisme classique, voire le plus radical minarchisme (Nozick). Chez ce dernier, il n'y a même pas de "filet de sécurité " pour les plus pauvres (ce serait imposer du "travail forcé » aux plus riches), alors que Hayek et Friedman ("impôt négatif ») admettent cette fonction de l'État. Mais ce dernier, essentiellement, ne contrôle que

\footnotetext{
${ }^{62}$ L'actuel régime chinois mélangeant ce système inefficace avec l'efficace mode $4 . .$.
} 
l'absence de violence et de fraude dans les transactions individuelles. Un simple "veilleur de nuit ", qui autrement "laisse faire ". Les rawlsiens refusent d'appeler cet arrangement institutionnel "libéral », ce qui me paraît excessif (alors que je suis prêt à le faire eu égard au mode de production précédent : il y a un lien fort entre la tradition libérale et l'idée d'État limité, mais non nul). Mais c'est un système capitaliste, au sens marxien que retient Rawls, à savoir un système où les grands moyens de production sont la propriété d'une minorité de "riches »: une oligarchie du capital. En cela, elle ne garantit pas que les libertés des citoyens ne soient pas simplement formelles, les très riches pouvant influer considérablement la vie politique, et elle est compatible avec d'immenses inégalités, nullement justifiées par le mécanisme des incitations. Le PD n'y a aucune place.

$5^{\circ}$ Un système capitaliste assorti d'un État-Providence. Son idéologie est souvent pensée en termes utilitaristes, ou "welfaristes" (Welfare State) et paternalistes, car sa finalité peut être interprétée en termes de maximisation du bonheur collectif, mais il peut être fondé sur une philosophie républicaine, qui insiste sur la participation de tous les citoyens à la décision politique et aux services publics, ou sur une vision sociale-démocrate, issue de Bernstein, avec une forte insistance sur le rôle des syndicats, allant jusqu'à la cogestion à l'allemande. Rawls préfère ce système aux précédents, mais tient qu'il ne permet pas de sortir de l'oligarchie capitaliste, de réaliser 
vraiment le PD et de donner toute leur "valeur » aux libertés de base. Les " très riches » ont toujours la possibilité d'influencer la vie politique pour de mauvaises raisons.

$6^{\circ}$ Le socialisme libéral, ou « de marché ", qui a les faveurs de Rawls, avec le suivant (7). L'État possède les grands moyens de production, et les entreprises sont dirigées soit par des fonctionnaires aux revenus confortables mais limités, ou par des personnes élues par les travailleurs concernés (autogestion, conseils ouvriers). Elles échangent leurs produits sur des marchés concurrentiels, sans monopoles: il peut y avoir plusieurs compagnies dans le même domaine, possédées par l'État mais en concurrence.

$7^{\circ}$ La "property-owning democracy " (démocratie de propriétaires), idée esquissée en particulier par l'économiste britannique James Meade, et qui semble avoir en fin de compte été préférée par Rawls au mode de production socialiste libéral ${ }^{63}$. Il s'agit d'une économie de marché fondée sur la propriété privée (ce qui n'empêche pas la copropriété, les coopératives), mais sans oligarchie capitaliste: le capital est largement dispersé, dans la logique de la démocratie. II ne s'agit pas seulement de la participation des travailleurs-actionnaires à la propriété de "leur » entreprise, ce qui serait pour eux un facteur de vulnérabilité, mais de la possession par chaque citoyen d'une part diversifiée du capital global. Elle peut prendre la forme d'une

${ }^{63}$ JF, Part. IV. 


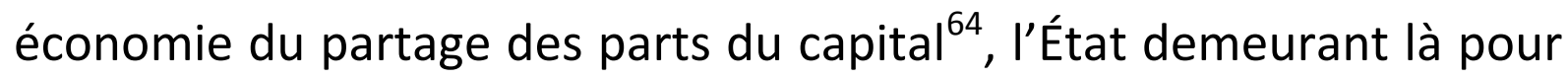
éviter tout drame social, dû par exemple à une faillite de l'entreprise, événement inévitable dans une économie ouverte de marché. La sévérité de Rawls envers le modèle 5 est étonnante. Lui opposer deux utopies, ou même une seule (le mode 7) ne peut se faire que si l'on donnait plus de précisions sur la manière dont le modèle utopique pourrait fonctionner, ainsi que sur la période de transition qui est censée y mener (imposition forte des successions) ${ }^{65}$. Cela reste à faire. De ce point de vue, l'utopie du revenu universel semble « moins » utopique.

De tous ces systèmes, notons que seuls les systèmes 2,4 et 5 ont existé ou existent (avec des variantes et des intersections). Que 1 et 3, qui prétendent pouvoir se passer de l'État ou du marché, sont des utopies irréalistes. Que seuls 3, 4, 5, 6 et 7 sont des économies de marché. Que Rawls considère que seuls 6 et 7 (et non 5, encore capitaliste) sont susceptibles de réaliser les principes de justice. Comme ils n'ont pas été expérimentés, mais qu'ils acceptent le marché et l'État, ce sont des "utopies réalistes ». En revanche, seuls 4 et 5 ont été ou sont à l'heure actuelle réalisés sans être des catastrophes absolues. (Les modes de production des purs chasseurs-

\footnotetext{
${ }^{64} J F$, § 20. Van Parijs (op.cit.) soutient qu'un revenu de base durant toute la vie est préférable à une dotation en capital à l'âge adulte. Voir la critique du revenu de base par Jon Elster : "Comments on van der Veen and Van Parijs ", Theory and Society, vol. 15, n5, 1986. ${ }^{65}$ Voir la critique sévère de Jon Elster, "Comments on Krouse and MacPherson ", Ethics, Vol. $97, N^{\circ} 1,1986$. Les effets pervers de telles amples réformes sont à craindre. Rawls n’y est pas sensible, et c'est dommage.
} 
cueilleurs (prétendu "communisme primitif»), et ceux que Marx appelle "asiatique », esclavagiste et féodal, et qui ne figurent pas dans la liste, ont pour l'essentiel disparu.)

Le mode de production du "socialisme de marché », utopie qui a plusieurs variantes, ne me paraît pas assez libéral (contra Rawls). Faudrait-il interdire les créations d'entreprises privées ? Et, malgré certains succès en Europe, n'est-il pas imprudent de confier la gestion d'une grande part des moyens de production à des fonctionnaires ? Non qu'il faille, à la manière des libertariens, cultiver une véritable suspicion a priori contre les agents de l'État, analogue à la suspicion des anti-libéraux contre tous les entrepreneurs privés, mais ne vaut-il pas mieux leur réserver une fonction de contrôle (au sens français), dans le cadre d'une saine division du travail ? Le socialisme de marché interdit a priori trop d'usages du droit de propriété, lequel, sans être du tout un "droit naturel », présente des avantages : on prend plus soin de ce qui nous appartient (même dans une copropriété) que de ce qui appartient à tous (propriété publique) $\left(\right.$ Aristote $^{66}$ ); et la propriété permet la «libéralité », le don ${ }^{67}$, l'échange, le prêt, le louage, le legs, l'hypothèque, le gage, la responsabilité pour les accidents, toutes formes d'interactions sociales qui ne manquent pas de mérites, dans la cadre de la coopération sociale si chère à Rawls. Bien sûr, il y a analytiquement

\footnotetext{
${ }^{66}$ Pol., II, 1261 b35 ; et saint Thomas, ST, II Ilae, qu. 66 (De furto et rapina).

67 Comme Jean-Jacques l'explique à Émile, l'Émile, II, "pour donner, il faut avoir » (Rousseau, CEuvres complètes, 3, Seuil, p. 67).
} 
dans l'idée de propriété celle d'exclusion d'autrui. Mais aussi celle d'usage possible par autrui s'il est consenti par le, la ou les propriétaires. Ce n'est pas l'institution de la propriété qui est " égoïste/exclusiviste », explique Aristote, c'est la nature humaine ${ }^{68}$ : autant "phileïn eauton » est naturel, autant la "philautia » est un excès : « tout pour moi ! ». L'amitié, vertu majeure, incite à partager, mais on ne partage que ce que l'on a, ce que l'on a le droit de posséder. Avec le christianisme, avec saint Ambroise, avec saint Thomas, c'est la charité qui jouera le rôle de la "libéralité » aristotélicienne. Bien sûr, Rawls a protégé le droit élémentaire de propriété, la "propriété personnelle » (dont on suppose qu'elle couvre le logement), dans le Premier Principe, mais pas la propriété des moyens de production (ce qui produit du fructus), et il est assez naturel que de penser que dans la $\mathrm{PO}$, seule la première sera défendue mordicus. Mais autant il peut être satisfaisant d'inciter les travailleurs à fonder des coopératives, autant il peut paraître dangereux d'avoir à interdire l'entreprise privée et le pouvoir des entrepreneurs au profit de celui des fonctionnaires.

Demeure selon moi le choix entre le capitalisme à ÉtatProvidence, parfois au bord de la faillite, et l'utopie de la "démocratie de propriétaires ». On peut bien entendu se satisfaire de l'État-Providence et de ses défauts, étant donné ses réussites locales indéniables depuis 1945. Mais si l'on désire l'améliorer, faut-il

${ }^{68}$ Pol., II, 1263 b. 
le faire dans le sens du socialisme de marché, sans liberté personnelle d'entreprendre et d'embaucher, ou dans celui de la démocratie de propriétaires $?^{69}$ Je ne suis pas le seul à préférer la seconde " utopie réaliste " à la première ${ }^{70}$, qui me paraît devoir s'éloigner du libéralisme en donnant trop de puissance économique à l'État propriétaire et entrepreneur (pas toujours pour le bien commun). La démocratie de propriétaires a des allures plus rousseauistes, si l'on se rappelle qu'en même temps qu'il faisait du premier performatif "Ceci est à moi!» le péché originel de la créature humaine, il mettait la même propriété dans l'ordre des valeurs conventionnelles mais "sacrées", peut-être même plus importante que la liberté, dans l'article de l'Encyclopédie "Économie politique ${ }^{71}$. Mais Rousseau souhaitait un système nettement moins inégalitaire que celui de son temps, les riches ne devant pas avoir de quoi s'acheter un pauvre, et les pauvres n'ayant pas besoin de se vendre. Démocratiser le capital a par ailleurs l'avantage de se couler dans la dynamique de la démocratisation, génialement repérée par Tocqueville, et de ne pas pour autant mettre en cause les très positifs avantages théoriques et pratiques du marché, mises en évidence, depuis Smith, par des Ricardo, des Bastiat, des Pareto, des Hayek, des

\footnotetext{
${ }^{69}$ Au sens de Meade et de Rawls, et non seulement au sens restreint que lui avait donné Mrs Thatcher (propriété de son logement).

${ }^{70}$ Alan Thomas, Republic of Equals, Predistribution and Property-Owning Democracy, Oxford, 2017.

${ }^{71}$ CEuvres, III, Gallimard, 1964. Le Projet pour la Corse indique qu'il est souhaitable que la propriété de l'État soit la plus grande possible, et celle des particuliers la plus petite, ce qui est plus « socialiste ».
} 
Arrow, etc. Une utopie susceptible de réconcilier critiques du capitalisme et admirateurs du marché comme ordre spontané capable de mobiliser des milliards d'informations dispersées et d'inciter à l'innovation et à l'amélioration des produits, sans tomber dans l'utopie critiquable de l'autosuffisance dudit marché sacralisé, lequel n'aurait pas besoin de limites, de régulations, de contrôles : on se demande bien pourquoi, car toute institution humaine en nécessite, en dépit de Hobbes. Que l'État ne soit pas lui-même un parangon de vertu et que les bureaucrates aient des intérêts propres qui ne soient pas nécessairement alignés sur les intérêts collectifs (difficiles par ailleurs à mettre en évidence) est cela dit vrai, et à prendre en compte: nouvelle instance du Quis custodiet ipsos custodiet? de Juvénal, mais que les philosophes n'ont pas tardé à appliquer à Platon. Le processus sans fin de la démocratie libérale et délibérative est lui-même fondé sur l'idée des contrôles et des équilibres mutuels des autorités; il n'y a donc pas de raison de penser que tous les fonctionnaires sont par essence incompétents, corrompus et non contrôlables. Et enfin, utopie permettant d'espérer une société où personne ne soit laissé de côté, ne perde les bases sociales du respect de lui-même et les moyens de réaliser peu ou prou ses projets rationnels dans le cadre de règles justes auxquelles il ou elle pourrait donner son accord réfléchi et les intégrer à ses fins. Une société solidaire de propriétaires moins enclins à l'envie, car satisfaits de leur part, et une société de coopération dans l'avantage 
réciproque, où des personnes accomplies puissent entretenir des relations sociales riches, et produire par leur coopération libre des œuvres communes dont elles pourraient être toutes fières. On peut cependant douter de la réalisabilité de cette " utopie réaliste », et la considérer comme un simple idéal régulateur, incitant à diminuer les excès de richesse et de pauvreté patrimoniales. Le principe de différence, moins important que les deux principes qui le précédent lexicalement, n'en demeure pas moins un principe philosophiquement fondé et qui n'a pas disparu des théories de la justice valant la peine d'être soumises à la réflexion critique. II représente l'une des manières les plus précises que l'on ait proposées pour interpréter le principe de fraternité ${ }^{72}$.

Alain Boyer

Sorbonne Université

${ }^{72} T J, \S 17$, par. $10-11$. 